Proceedings of the 15th Czech and Slovak Conference on Magnetism, Košice, Slovakia, June 17-21 2013

\title{
Structure and Properties of Composites Based on Mixed Morphology of Ferromagnetic Particles
}

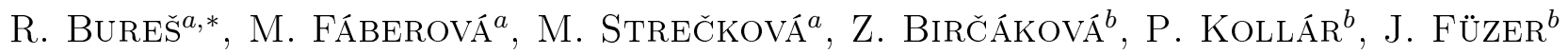 \\ ${ }^{a}$ Institute of Materials Research, Slovak Academy of Sciences, Watsonova 47, 04001 Košice, Slovakia \\ ${ }^{b}$ Institute of Physics, Faculty of Sciences, P.J. Šafárik University, Park Angelinum 9, 04154 Košice, Slovakia
}

Application potential of the soft magnetic composites increases with improvement of their functional properties. In addition to the magnetic properties, the mechanical properties of these materials are essential. The aim of this work was to investigate mechanical properties and their dependence on morphology of the ferromagnetic particles. Model composite based on Somaloy ${ }^{\circledR}$ and Vitroperm ${ }^{\circledR}$ powder was prepared using conventional powder metallurgy. The DC magnetic properties of composites based on two types of ferromagnetic particles with different shapes and structures are discussed in the relation with mechanical properties.

DOI: 10.12693/APhysPolA.126.140

PACS: 81.05.-t, 75.50.Bb

\section{Introduction}

Soft magnetic composites (SMC) are materials based on ferromagnetic powder particles insulated-bonded by organic, inorganic or hybrid coating layer [1]. Low eddy current losses, 3D-flux carrying capability and costefficient production of 3D-net shaped components by the PM process are main features, which are offered by this technology [2]. In addition to the magnetic properties, knowledge about the mechanical properties of these materials is essential, if information about the resistance to mechanical stresses is needed [3].

The aim of this work was to continue of our previous investigations of high frequency magnetic properties of composite material based on the mixture of two ferromagnets: insulated polycrystalline iron particles and nanocrystalline Fe-based alloy [4]. Another goal of this work was to investigate the changes of mechanical properties of the composite in dependence on morphology of ferromagnetic particles.

\section{Experimental materials and methods}

Somaloy ${ }^{\circledR} 700$ premix powder (S) supplied by Höganäs AB, Sweden and Vitroperm ${ }^{\circledR} 500$ flaky powder (VPM) supplied by Vacuumschmelze, GmbH \& Co. KG, Germany were used. Powders were mixed in appropriate ratio $(5,10,20,30,40$ and $50 \mathrm{wt} . \%$ of VPM) and uniaxially cold pressed at pressure of $800 \mathrm{MPa}$ into the shape of cylinder $(d=10 \mathrm{~mm}, h=3 \mathrm{~mm})$, bar $(4 \times 5 \times 20 \mathrm{~mm})$ and ring $\left(d_{\text {out }}=24 \mathrm{~mm}, d_{\text {in }}=17 \mathrm{~mm}, h=3 \mathrm{~mm}\right)$. Green compacts were heat treated at temperature of $530{ }^{\circ} \mathrm{C}$ during 1 hour in air or Ar atmosphere.

Density of the composites was measured by He pycnometry. Elastic constant was measured by impulse excitation technique according to ASTM E1876. Mechanical properties were tested by measurement of the Vickers hardness HV10 and by measurement of transverse

*corresponding author; e-mail: rbures@imr.saske.sk rupture strength TRS by three point bending test. Microstructures and fractures were investigated by light optical microscopy LOM and scanning electron microscopy SEM. DC hysteresis loops were measured by a fluxmeter based hysteresisgraph at maximum induction of $0.2 \mathrm{~T}$.

\section{Results and discussion}

Somaloy particles are well insulated by unbroken original coating (Fig. 1a). In Somaloy, pores are located mainly at the connection point of three or more initial particles. Resin bonded VPM particles show clusters of flat lying particles (Fig. 1b). Flaky VPM-particles are located as a quasi layer on S-particles (Fig. 1c).

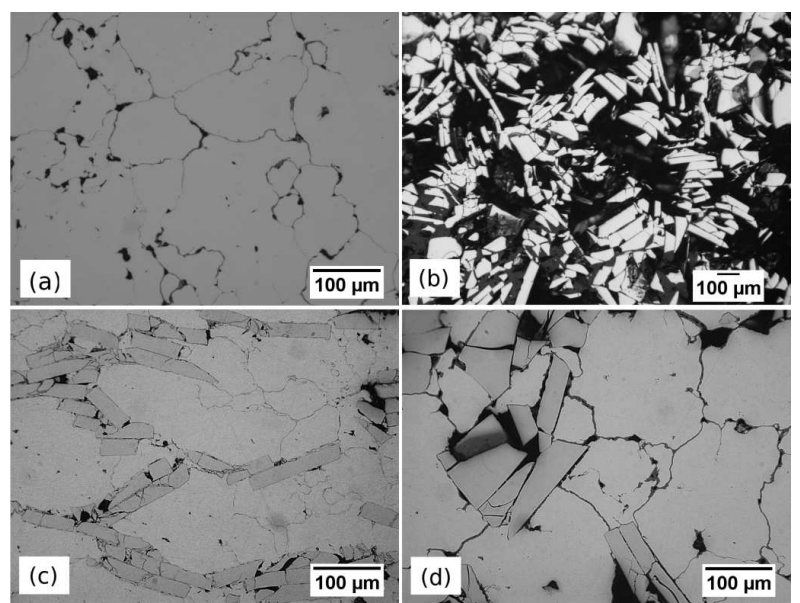

Fig. 1. LOM microstructure of the composites, (a) Somaloy, (b) Resin bonded VPM, (c) S30VPM, (d) S50VPM.

Flaky morphology of VPM, their deformation and cracking improve densification of powder mixture to the ratio 10 wt.\% of VPM in composite (Fig. 2). Increase of VPM fraction leads to more frequent cracking of flaky particles (Fig. 1c). At 40-50 wt.\% of VPM, the Sparticles and their insulation layer is plastically deformed up to eventual failure (Fig. 1d).

Density measured by He pycnometry involves closed porosity of the composite. The values of total porosity, calculated from size of bar samples, include close 


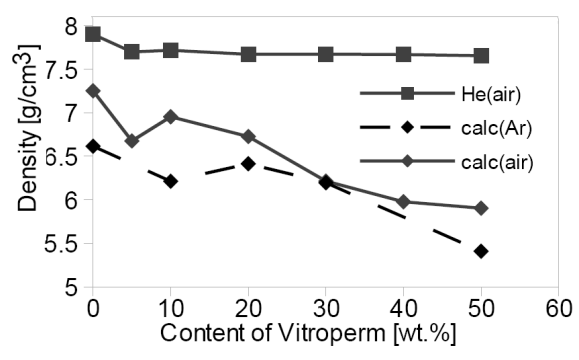

Fig. 2. Density vs. VPM fraction.

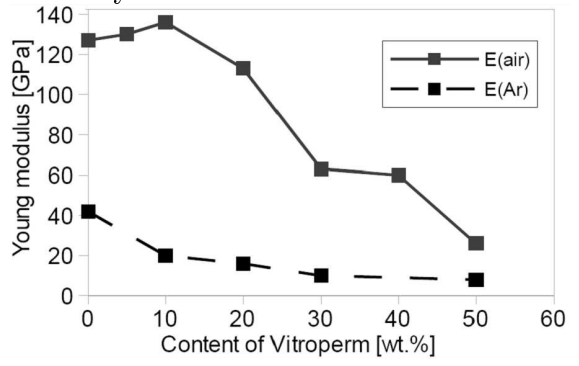

Fig. 3. E vs. VPM fraction.

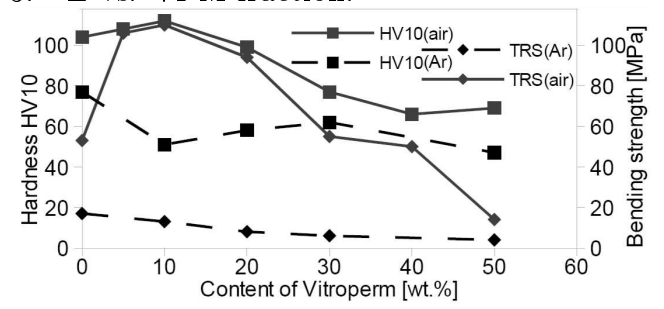

Fig. 4. Mechanical properties vs. VPM fraction.
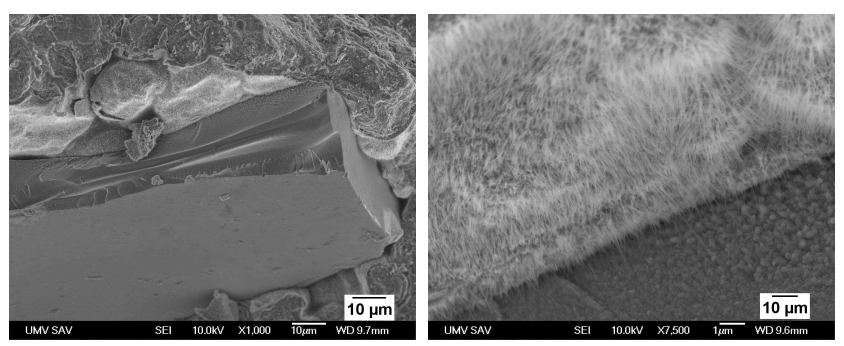

Fig. 5. Interperticle connection of S and VPM (SEM - fracture), (left) Somaloy-Vitroperm particles, (right) Oxide-bond connection.

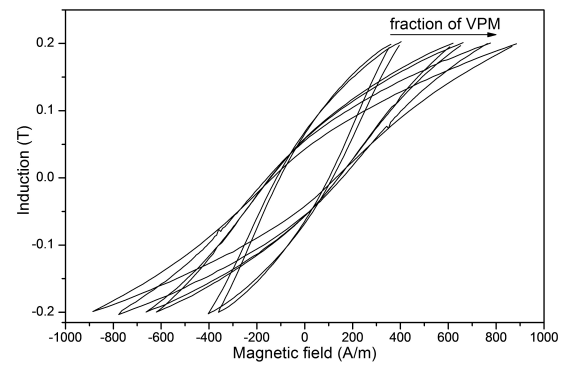

Fig. 6. DC hysteresis loops at maximum induction of $0.2 \mathrm{~T}$ of samples with $5 \%, 10 \%, 20 \%, 30 \%, 40 \%$ and $50 \% \mathrm{VPM}$ and open pores (Fig. 2). Density is the main, but not the only parameter, which affects mechanical properties. Hardness, plastic and elastic properties achieve the highest value at $10 \mathrm{wt} . \%$ of VPM (Fig. 3, 4). Improvement of the mechanical properties is caused by oxide based SVPM interparticle connections after heat treatment in air (Fig. 5). Increase of VPM fraction as well as heat treatment in Ar atmosphere decrease mechanical properties of this model composite.

DC hysteresis loops at maximum induction of $0.2 \mathrm{~T}$ of samples with $5 \%, 10 \%, 20 \%, 30 \%, 40 \%$ and $50 \%$ VPM are shown in Fig. 6. It can be seen that DC peak permeability (ratio of maximum induction to maximum magnetic field) decreases with VPM content. It is caused by the decrease of the density and simultaneous increase of inner demagnetizing fields of these VPM flakes, which are oriented non-parallel to the induction vector in the sample.

Further magnetic properties of described composites are also discussed in [5].

\section{Conclusions}

Mechanical properties of SMC could be improved by mixing particles of two suitable morphologies. Proper morphology and fraction of flaky powder particles could lead to higher bending strength, hardness and elastic properties of the composite.

For the decrease of total losses with VPM content it is required the parallel orientation of VPM flakes in composite material with higher possibility of domain wall displacement.

\section{Acknowledgments}

This work was supported by the Slovak Research and Development Agency through project APVV-022210 MAGCOMP, by the projects, nanoCEXmat I, ITMS 26220120019 and nanoCEXmat II, ITMS: 26220120035 supported by the Operational Program "Research and Development" financed through European Regional Development Fund and by the Scientific Grant Agency of the Ministry of Education of Slovak Republic and the Slovak Academy of Sciences, projects Nos. VEGA $1 / 0861 / 12$, No. $1 / 0862 / 12$ and $2 / 0155 / 12$. Special thanks to Höganäs AB Sweden for providing Somaloy ${ }^{\circledR}$ 700 powder and to Mr. M. Vitovský, Vacuumschmelze, GmbH \& Co. KG, Germany for providing Vitroperm ${ }^{\circledR} 500$.

\section{References}

[1] J.A. Bas, J.A. Calero, M.J. Dougan, J. Magn. Magn. Mater. 254-255, 391 (2003).

[2] L. Hultman, O. Andersson, $M P R$, July/August, 26 (2010).

[3] S. Gimenez, T. Lauwagie, G. Roebben, W. Heylen, O. Van der Biest, J. Alloy Compd. 419, 299 (2006).

[4] J. Füzerová, J. Füzer, P. Kollár, L. Hegedüs, R. Bureš, M. Fáberová, IEEE Trans. on Mag. 48, 1545 (2012).

[5] Z. Birčáková, P. Kollár, V. Vojtek, R. Bureš, M. Fáberová, Acta Phys. Pol. A 126, 114 (2014). 